\title{
Auf der Suche nach einem europäischen Konsens - zur Rechtsprechung des Europäischen Gerichtshofs für Menschenrechte
}

\author{
Angelika Nußberger"
}

\section{A. Facettenreichtum des Themas „Kollektivität“}

Sie haben für die Assistententagung in Hamburg das Thema „Kollektivität“ gewählt und sich mit kollektiven Akteuren und Handlungsformen befasst. Sie sehen Kollektivität als Motor dogmatischer Innovation an, fragen nach den Einstandspflichten aus Solidarität, spüren der Tyrannei der Mehrheit und der Macht der Minderheit nach. Auch die europäische Perspektive scheint in Ihren Vorträgen auf, wenn Sie über die Öffentlichkeitsbeteiligung im Recht der EU und ihre Einwirkungen auf das deutsche Verwaltungsrecht oder über den Dialog mit repräsentativen Verbänden und der Zivilgesellschaft als Mittel zur Stärkung der Demokratie in der Europäischen Union nachdenken.

Nun ist wohl ein Kollektiv, gleich ob ein Verband, eine NGO, eine Bürgergemeinschaft oder auch eine Gruppe von Staaten, nur dann ein Kollektiv und keine Ansammlung von Einzelnen, wenn es sich über gemeinsame Werte definieren kann. Diese müssen zumindest nach innen eine identitätsstiftende Wirkung haben, mögen vielleicht auch nach außen zu einer Abgrenzung führen. Wer sich für eine bestimmte Sache einsetzt, für eine bestimmte Gruppe von Menschen spricht, bestimmte Interessen vertritt, unterscheidet sich von „den Anderen“. Sie sind dem Interessenausgleich in dem spannungsreichen Feld Individuum - Kollektiv - Gesamtgemeinschaft, vor allem im staatlichen Rahmen nachgegangen. In meinem Festvortrag gestehen Sie mir zu, über den Staat hinauszugreifen und den Blick auf Europa zu werfen. Ich will mit Ihnen gemeinsam fragen, wie Europa die Werte definiert, über die es sich dann selbst definiert.

\section{B. Europa als „Kollektiv“}

Blicken wir auf Europa, verschiebt sich die Perspektive. Es ist ein großes Kollektiv, das 800 Millionen Menschen umfasst. Es ist ein Kollektiv, dass eine Vielzahl von Untereinheiten aufweist. Auf der Landkarte sehen wir üblicherweise die Farben der einzelnen Staaten als deutliches Unterscheidungskriterium, wobei die Größe der

* Bei dem nachfolgend abgedruckten Text handelt es sich um die auf dem 52. Deutschen Assistententag zum Thema „Kollektivität“ in Hamburg am 15.3.2012 gehaltene Festrede. Die darin enthaltene Analyse der Entscheidung Marckx v. Belgien einerseits und ABC v. Irland andererseits ist Teil des beim Verlag Duncker und Humblot unter dem Titel „Der Wandel der Grundrechte“ erschienenen Beitrags zur Festschrift für Klaus Stern (Michael Sachs, Helmut Siekmann (Hg.), Der grundrechtsgeprägte Verfassungsstaat. Festschrift für Klaus Stern zum 80. Geburtstag, Berlin 2012,S. 117-138). Der Text gibt ausschließlich die Meinung der Verfasserin wieder und bindet den Europäischen Gerichtshof für Menschenrechte in keiner Weise. 
Staaten stark variiert - von San Marino mit knapp 30.000 Einwohnern bis zur Russischen Föderation mit 142 Millionen. Mit Schraffuren können Sie über den Länderfarben das Europa der Regionen markieren. Oder aber Sie können die Staaten in noch größeren Einheiten zusammenfassen: die nordeuropäischen Staaten, die baltischen Staaten, die ex-sowjetischen Staaten, die südeuropäischen Staaten. Europa ist also ein kollektives Ganzes, das sich aus vielen unterschiedlichen einzelnen kollektiven Einheiten zusammensetzt.

Wie findet nun in einem derart komplexen Gebilde Identitätsbildung statt? Warum vermag sich ein Europäer als Europäer zu fühlen?

Man mag den entsprechenden identitätsstiftenden Wertsetzungen auf der Grundlage der Geschichte, der Soziologie, der Psychologie nachgehen. Das ist aber nicht unser Werkzeug. Als Juristen fragen wir danach, auf der Grundlage welcher Verfahren Werte festgelegt und für verbindlich erklärt werden, welche Akteure daran auf welche Weise beteiligt sind, ob der Prozess so gestaltet ist, dass er die Wirklichkeit adäquat widerspiegelt. Spannend ist vor allem auch, wie Wertewandel stattfindet.

Auf der staatlichen Ebene können wir von der Verfassung ausgehen. Verfassungen enthalten, nicht nur, aber vor allem im Grundrechtsbereich, Wertsetzungen. Diese gilt es in einem komplexen Prozess, eingebunden in das Wirken der verschiedenen staatlichen Gewalten, umzusetzen und zugleich weiterzuentwickeln. Wenn Sie das Familienrecht der 50er Jahre mit dem Familienrecht der Gegenwart vergleichen, sind unterschiedliche Wertsetzungen evident, auch wenn der Obersatz „Ehe und Familie stehen unter dem besonderen Schutz der staatlichen Ordnung“ unverändert geblieben ist; die Entwicklung hat im Wechselspiel zwischen innovativer Gesetzgebung und verfassungsrechtlicher Interpretation stattgefunden.

Nun will ich dieser Frage auf der europäischen Ebene nachgehen. Mein Thema lautet „Auf der Suche nach einem europäischen Konsens“. Eine gemeinsame Verfassung haben die 47 Staaten des Europarats nicht. Vielmehr befinden wir uns hier auf der Ebene des Völkerrechts. Wir haben nicht mehr - oder aber nicht weniger - als eine internationale Organisation, den Europarat, eine Vielzahl von Verträgen, von denen der entscheidende die Europäische Menschenrechtskonvention ist, und einen Gerichtshof, der dazu aufgerufen ist, diesen völkerrechtlichen Vertrag in Einzelentscheidungen mit für die Staaten bindender Wirkung auszulegen.

\section{Wertsetzung auf der Ebene der Europäischen Menschenrechtskonvention}

Die Europäische Menschenrechtskonvention definiert Mindeststandards, Menschenrechte, die allen Menschen zustehen, mit dem Pathos der Französischen Revolution gesprochen „Wahrheiten für alle Zeiten und alle Länder“. Nun mag das im Grundsatz stimmen, wenn Sie Postulate wie „Niemand darf der Folter oder un- 
menschlicher oder erniedrigender Behandlung oder Strafe unterworfen werden " lesen. Allerdings beginnen sogleich schon die Unsicherheiten. Was ist „unmenschlich“? Denken Sie an den Satz „Prügel haben noch niemandem geschadet“, der im kollektiven Gedächtnis der in den 50er Jahren Geborenen noch vorhanden sein dürfte heute würde man das, was früher „allzumenschlich“, „normal“, war, als „unmenschlich " bezeichnen und entsprechenden Erziehungspraktiken einen menschenrechtlichen Riegel vorschieben. Wie aber kommen wir darauf? Woher nehmen wir die Gewissheit, dass nicht mehr richtig ist, was jahrzehntelang, manches Mal jahrhundertelang als richtig angesehen worden ist? Wie finden wir, wie definieren wir die sich neu herausbildenden Werte?

Auf europäischer Ebene ist der Europäische Gerichtshof für Menschenrechte, sind die 47 Richterinnen und Richter, die aus 47 verschiedenen Ländern kommen, dazu aufgerufen, die Europäische Menschenrechtskonvention als „lebendiges Instrument“ zu verstehen und die in der Konvention festgehaltenen Werte dem gesellschaftlichen Wandel in Europa entsprechend auszulegen. Aber wie? Mit Tyrer v. Vereinigtes Königreich wurde im Jahr 1978 ein Anfang gemacht. Die Prügelstrafe wurde aufgrund von Art. 3 EMRK als Verstoß gegen Menschenrechte erkannt. Im Rückblick wird diesem Ausspruch niemand entgegentreten. Aber wie sind die aktuellen Fragen zu beurteilen? Widerspricht es den Menschenrechten in Europa, wenn Inzest zwischen Bruder und Schwester strafbewehrt ist, wenn man deswegen ins Gefängnis muss, nicht aber bei einer homosexuellen Beziehung innerhalb der Familie? Ist das etwa in Frankreich bestehende Verbot für diejenigen, die in einer gleichgeschlechtlichen Beziehung leben, das Kind des Partners zu adoptieren, ein Verstoß gegen die Menschenrechtskonvention? Wie weit gehen die Verpflichtungen der Staaten - müssen sie neben natürlichen Personen auch juristischen Personen Prozesskostenhilfe gewähren, müssen sie etwa - so die Frage in einem gerade gegen Deutschland anhängigen Verfahren - auch einer aus Peru stammenden, in Konkurs geratenen Gesellschaft finanzielle Unterstützung zukommen lassen, wenn sie in Eigentumsfragen gegen eine deutsche Gesellschaft klagt? Ist Erziehungsgeld ein menschenrechtliches „must“? Wie steht es mit Kriegsdienstverweigerung - besteht auch darauf ein europaweit einklagbares Recht?

Der Europäische Gerichtshof für Menschenrechte ist dazu aufgerufen, in immer neuen Fallkonstellationen mit Blick auf potentielle Konventionsverletzungen erstens zu prüfen, ob ein bestimmtes Grundrechtsverständnis fortbesteht oder sich eine neue Anschauung herausgebildet hat und ob dann zweitens, in der Folge, der einzelne Staat auf der Grundlage der EMRK wegen einer Menschenrechtsverletzung zu verurteilen ist, wenn er sich der Entwicklung - dem menschenrechtlichen Zeitgeist, wenn man so will - entgegenstellt. Wie geht der Gerichtshof auf der Suche nach dem eu- 
ropäischen Konsens vor? Und wie viel Raum belässt er abweichenden Überzeugungen und Anschauungen?

\section{Grundlagen der Argumentation}

Die Argumentation des Gerichtshofs bindet zwei zentrale Elemente ein. Zum einen gilt es festzustellen, ob ein Konsens besteht, („existence of a consensus“), zum anderen ist - abhängig davon - der so genannte Ermessensspielraum der Staaten, der „margin of appreciation“ auszuloten.

\section{I. „Konsens“}

Nun ist mit Blick auf die Existenz eines Konsenses zu differenzieren. Zu unterscheiden ist zwischen „historischem Konsens“ der Gemeinschaft der Staaten, „individualstaatlichem Konsens“ und „lebendigem Konsens“.

Der „historische Konsens“ verweist auf das, was die Vertragspartner bei der Ausarbeitung der Konvention intendiert haben und ist vergleichsweise einfach festzustellen. Man braucht nur auf die Archive zurückzugreifen und Einsicht in die Verhandlungsprotokolle zu nehmen. In den travaux préparatoires findet sich so mancher Hinweis. Der Gerichtshof legt darauf allerdings offensichtlich keinen großen Wert. Von Ausnahmen abgesehen - etwa in der Bankovic-Entscheidung - sieht der Gerichtshof den in den 50er Jahren gefundenen Konsens für nicht relevant an. So sagt er etwa in der Entscheidung Loizidou explizit, wenn es um die Abgrenzung der Jurisdiktion des Gerichtshofs geht, die Bestimmungen der Konvention

"cannot be interpreted solely in accordance with the intentions of the authors as expressed more that forty years ago."

Auch über den jeweiligen individualstaatlichen Konsens oder Dissens, so wie er den Vorbehalten und Erklärungen zur Konvention zu entnehmen ist, die eine Vielzahl von Staaten zum Zeitpunkt der Ratifikation abgegeben haben, geht der Gerichtshof vergleichsweise leichtfüßig hinweg, man denke nur an die Urteile in den Fällen Belilos v. Schweiz oder Loizidou v. Türkei, in denen der Gerichtshof die Mitgliedsstaaten entgegen ihrem ausdrücklich erklärten Willen als an die Konvention gebunden ansieht.

Weder der historische noch der individualstaatliche Konsens sind also ausschlaggebend für die Interpretation der Konvention. Relevant ist dagegen der „lebendige Konsens“, worunter die jeweils geänderten Rechtsanschauungen in den einzelnen Mitgliedsstaaten zu verstehen sind. Diese sind allerdings nicht einfach festzustellen. Der Gerichtshof nimmt dabei einen Kunstgriff zur Hilfe und geht davon aus, dass sich dieser Konsens in den jeweils geltenden nationalen Gesetzen ablesen lassen müs- 
se. Damit wird Rechtsvergleichung, die Gegenüberstellung der jeweils in den verschiedenen Mitgliedsstaaten geltenden Regelungen - anders als etwa in der nationalstaatlichen Verfassungsgerichtsbarkeit - zu einer entscheidenden Komponente bei der Auslegung der Konvention.

\section{Marckx v. Belgien - Mehrheitsvotum für den Wandel}

Im Folgenden soll am Beispiel der berühmten Marckx-Entscheidung des Gerichtshofs gezeigt werden, wie der europäische Konsens im Detail herausgearbeitet wird. In dem Fall hatte eine belgische Frau es als Verletzung ihrer Rechte sowie der Rechte ihres Kindes nach Art. 8 und 14 EMRK angesehen, dass das unehelich geborene Mädchen gegenüber ehelich geborenen Kindern in vielerlei Weise benachteiligt wurde, dass sogar die Mutterschaft erst anerkannt werden musste und damit verwandtschaftliche Beziehungen zum Vater abgeschnitten waren.

Der Schutz der Familie ist ein zentrales Thema des verfassungsrechtlichen wie auch des internationalen Menschenrechtsschutzes. Das klassische Konzept, wonach die Familie die „natürliche Kernzelle der Gesellschaft“ ist und „Anspruch auf Schutz durch Gesellschaft und Staat“ hat, kann aber eine sehr unterschiedliche Bedeutung und Wirkung haben je nachdem, wie weit der Begriff „Familie“ verstanden wird.

Der Gerichtshof gesteht in der Entscheidung Marckx v. Belgien ein, dass zum Zeitpunkt der Ausarbeitung der Konvention Orientierungspunkt die „de-iure-Familie“ als Gemeinschaft zwischen einem verheirateten Paar und gemeinsamen Kindern war und daher eine „legitime“ von einer „illegitimen“ Familie abgegrenzt wurde:

"It is true that, at the time when the Convention of 4 November 1950 was drafted, it was regarded as permissible and normal in many European countries to draw a distinction in this area between the "illegitimate" and the "legitimate" family."2

Weiter führt der Gerichtshof aus, dass entsprechende Trennlinien über viele Jahre hin in einer großen Zahl von Vertragsstaaten gezogen worden seien; die Entwicklung zur Gleichheit sei langsam und die Bezugnahme auf die Konvention erst zu einem späten Zeitpunkt in Erwägung gezogen worden. Daher überrascht es auch nicht, dass die Kommission in den 60er Jahren Beschwerden gegen Regelungen, die nicht-eheliche Kinder etwa im Bereich des Erbrechts benachteiligten, ohne weitere Prüfung verwarf.

In der Entscheidung Marckx bricht der Gerichtshof nun aber mit diesem Ansatz und interpretiert den Begriff „Familie“ weit; erfasst sieht er all diejenigen, die de facto in einer Familiengemeinschaft zusammenleben. Damit verändert sich der Schutzbereich von Art. 8 der Konvention insbesondere im Zusammenspiel mit Art. 14 EMRK 
grundlegend, da Ungleichbehandlungen zwischen „klassischen Familien“ und anderen familienrechtlichen Formationen wie der Beziehung zwischen Mutter und nichtehelichem Kind damit grundsätzlich konventionsrechtlich verboten sind.

Bei dieser neuen - erweiternden - Interpretation wendet der Gerichtshof Interpretationsstrategien an, die über das klassische Muster, das sich für völkerrechtliche Verträge nach Art. 31 WVK bestimmt, in gewisser Weise hinausgehen. Zwar rekurriert er in der Entscheidung Marckx zunächst - und insofern ist es ein traditioneller Ansatz - auf den Wortlaut von Artikel 8 EMRK. Danach sieht er als entscheidend an, dass kein expliziter Unterschied zwischen einer „legitimen“ und einer „,illegitimen“ Familie gemacht werde. Außerdem stellt er auf das Wort „everyone“ ab. Habe „jede Person“ das Recht auf Achtung ihres Familienlebens, könnten insofern keine Unterschiede gemacht werden, zumal Artikel 14 den Rückgriff auf die „Geburt“ als diskriminierend brandmarke. Zu einem anderen Ergebnis hätte der Gerichtshof hier kommen können, hätte er Art. 8 EMRK im Zusammenhang mit Art. 12 EMRK interpretiert, wonach „Männer und Frauen im heiratsfähigen Alter ... das Recht [haben], eine „Ehe einzugehen und eine Familie zu gründen“, denn dies hätte man als Ausdruck des klassischen Familienkonzepts lesen können, bei dem die Eheschließung der Familiengründung vorausgeht.

Wichtig ist aber weniger, ob der Gerichtshof den Begriff Familie eng oder weit auslegt und wie er diese Auslegung am Wortlaut der Konvention festmacht. Relevant ist vielmehr die Tatsache, dass der Gerichtshof sich explizit dazu bekennt, die Konvention mit Blick auf den gesellschaftlichen Wandel zu interpretieren. Zur Begründung verweist er auf sein eigenes - erst wenige Monate zuvor geschaffenes - case law, konkret auf die Entscheidung Tyrer v. Vereinigtes Königreich, in der er die Konvention zum ersten Mal als „lebendes Instrument“ bezeichnet hatte. Die Annahme, die Konvention sei „lebendig“, sieht der Gerichtshof als allgemeine Charakterbestimmung der Konvention an und erhebt sie zum Programm.

Damit aber stellt sich die entscheidende Frage, wie der Wandel, den das „lebendige Instrument" abbildet, zu bestimmen sei. Die Argumentation des Gerichtshofs baut hierzu im Wesentlichen auf drei Säulen auf: auf dem Ergebnis einer rechtsvergleichenden Analyse, auf dem Verweis zu Entwicklungen im Völkervertragsrecht und auf Stellungnahmen des Ministerkomitees des Europarats und damit internationales soft law. Die entscheidende Passage des Urteils lautet:

"It is true that, at the time when the Convention of 4 November 1950 was drafted, it was regarded as permissible and normal in many European countries to draw a distinction in this area between the "illegitimate" and the "legitimate "family. However, the Court recalls that this Convention must be interpreted in the light of present-day conditions (Tyrer judgment of 25 April 1978, Series 
A no. 26, p. 15, para. 31). In the instant case, the Court cannot but be struck by the fact that the domestic law of the great majority of the member States of the Council of Europe has evolved and is continuing to evolve, in company with the relevant international instruments, towards full juridical recognition of the maxim "mater semper certa est". ${ }^{3}$

Interessant ist nun, wie der Gerichtshof im Einzelnen den Nachweis zu den Änderungen, die eine neue Interpretation rechtfertigen, führt. Die Rechtsentwicklung in den Vertragsstaaten des Europarats wird nicht im Einzelnen nachgewiesen; der Gerichtshof lässt den allgemeinen Verweis, der auch ein prognostisches Element enthält, insofern ausreichen.

Genauer befasst er sich mit der völkerrechtlichen Situation. Als relevant erachtet er die Brüssler Konvention vom 12.9.1962 „On the Establishment of Maternal Affiliation of Natural Children", die am 23.4.1964 in Kraft trat sowie die Konvention vom 15.10.1975 „On the Legal Status of Children born out of Wedlock“, die im Rahmen des Europarats ausgearbeitet wurde und am 11.8.1978 und damit unmittelbar vor der Entscheidung im Fall Marckx in Kraft trat. Allerdings waren an beide Konventionen zum Zeitpunkt der Entscheidung nur vier Staaten und damit ein Fünftel der Vertragsstaaten der Konvention gebunden. Belgien, gegen das die Beschwerde im Verfahren Marckx gerichtet ist, hatte die erste Konvention unterzeichnet, aber nicht ratifiziert, die zweite Konvention noch nicht einmal unterzeichnet. Trotz der geringen Ratifikationsdichte erkennt der Gerichtshof einen Konsens mit Blick auf die evolutive Entwicklung des Familienrechts in diesem Bereich. So führt er aus:

„However, this state of affairs cannot be relied on in opposition to the evolution noted above. Both the relevant Conventions are in force and there is no reason to attribute the currently small number of Contracting States to a refusal to admit equality between "illegitimate" and legitimate" children on the point under consideration. In fact, the existence of these two treaties denotes that there is a clear measure of common ground in this area amongst modern societies." 4

Schließlich weist der Gerichtshof noch auf die Resolution des Ministerkomitees von 1970 "On the social protection of unmarried mothers and their children", die den Vertragsstaaten eine Reihe von Hilfs- und Unterstützungsmaßnahmen für Mütter nicht-ehelicher Kinder empfiehlt. Eine Gleichbehandlung mit verheirateten Paaren mit Kindern wird aber nicht gefordert.

Dies bedeutet, dass an den Nachweis zum Rechtswandel, als dessen Spiegel die Konvention verstanden wird, keine allzu hohen Ansprüche gestellt werden. 
Ausgehend von diesem gewandelten Familienverständnis argumentiert der Gerichtshof, dass das belgische Recht, das ehelich und uneheliche geborene Kinder grundsätzlich unterschiedlich behandelt, gegen Art. 8 und Art. 14 EMRK verstoße. Belgien verweist zur Begründung seiner Gegenposition auf die gesellschaftlichen Moralvorstellungen, die nach dem Wortlaut von Art. 8 EMRK zur Einschränkung auch des Familienlebens berechtigten.

Der Gerichtshof weist diese Argumentation aber entschieden zurück:

"The Court recognises that support and encouragement of the traditional family is in itself legitimate or even praiseworthy. However, in the achievement of this end recourse must not be had to measures whose object or result is, as in the present case, to prejudice the "illegitimate" family; the members of the "illegitimate" family enjoy the guarantees of Article 8 (art. 8) on an equal footing with the members of the traditional family." 5

Das telos, das der Gerichtshof mit seinem Urteil verfolgt, ist durchaus sozialpolitischer Natur. Explizit verweist der Gerichtshof darauf, es gelte zu ermöglichen, dass sich das Familienleben einer unverheirateten Mutter mit ihrem Kind normal entwickeln könne.

Grundrechtsschutz findet, insbesondere wenn es nicht nur um die Abwehr staatlicher Eingriffe geht, immer in einem feinmaschigen Netz von Rechten und Pflichten statt; Einzelinteressen Betroffener können sich unter Umständen konträr gegenüberstehen. Dies hat der Gerichtshof bei der Auslegung der Konvention zu berücksichtigen. Bei der Entscheidung Marckx klingt diese Überlegung auch an, da es etwa im Bereich des Erbrechts nicht von der Hand zu weisen ist, dass die Zuerkennung von Rechten an nicht-eheliche Kinder eine Schmälerung der Rechte der ehelichen Kinder mit sich bringen kann. Der Gerichtshof sieht dies aber dennoch nicht als Grund, um die Schlechterstellung der nicht-ehelichen Kinder zu akzeptieren:

"Admittedly, the "tranquillity" of "legitimate" families may sometimes be disturbed if an "illegitimate" child is included, in the eyes of the law, in his mother's family on the same footing as a child born in wedlock, but this is not a motive that justifies depriving the former child of fundamental rights." 6

Wesentliche Grundlage für die Analyse des menschenrechtlichen status quo sind also rechtsvergleichende Untersuchungen mit Blick auf eine „normative Konsensbildung “. Wird eine Rechtsfrage in einer Mehrzahl von dem Europarat unterworfenen Rechtssystemen in ähnlicher Weise beantwortet, so kann dies ein Indiz dessen sein, 
was als grundrechtlich geboten angesehen wird. Dies ist das klare Credo des Gerichtshofs:

„The existence of a consensus has long played a role in the development and evolution of Convention protections...Consensus has therefore been invoked to justify a dynamic interpretation of the Convention (...)."”

Der margin of appreciation wird in der Folge zu einer Variable der Konvergenz der Rechtsordnungen: Je unterschiedlicher die Regelungen in den verschiedenen Rechtsordnungen sind, desto größer ist der Beurteilungsspielraum des nationalen Gesetzgebers. Umgekehrt gilt, dass dann, wenn die Regelungen in der Mehrheit der Staaten übereinstimmen, von einem eher engen margin of appreciation auszugehen ist.

In der Rückschau wird man zweifellos sagen können, dass die Entscheidung Marckx v. Belgien ganz überwiegend positiv aufgenommen worden ist, mehr noch, dass sie den Erfolg des europäischen Menschenrechtsschutzsystems auf der Grundlage der Konvention als eines „lebendigen“ Instruments entscheidend mitbegründet hat. Sie hat den „Nerv der Zeit“ getroffen und war Zeichen des Fortschritts, indem sie ermöglichte, ungerechtfertigte Ungleichbehandlungen aufzudecken und zu sanktionieren.

\section{E. Akzeptanzprobleme}

In den nachfolgenden Urteilen, die das europäische Schutzsystem weiterentwickeln, sind in der Regel dieselben Argumentationsmuster zu erkennen. Nicht bei allen Entscheidungen aber ist es so evident, in welche Richtung eine vom gesellschaftlichen Konsens getragene Menschenrechtsrechtsprechung zu gehen habe. Insbesondere erfährt der Gerichtshof oftmals deutlichen Widerspruch aus den Staaten, denen aufgrund einer in anderen Vertragsstaaten beobachtbaren Rechtsentwicklung eine Menschenrechtsverletzung angelastet wird, insbesondere, soweit dies besonders sensible und kontroverse innenpolitische Themen betrifft.

Dies gilt beispielsweise für das Urteil Hirst v. Vereinigtes Königreich. Dabei geht es um die Auslegung von Art. 3 des Ersten Zusatzprotokolls zur EMRK, wonach die Vertragsparteien verpflichtet sind, in angemessenen Zeitabständen freie und geheime Wahlen unter Bedingungen abzuhalten, welch die freie Äußerung der Meinung des Volkes bei der Wahl der gesetzgebenden Körperschaften gewährleisten. In der Entscheidung Hirst v. Vereinigtes Königreich hat die Große Kammer des Gerichtshofs diese Bestimmung dahingehend ausgelegt, dass ein Wahlrecht, das Strafgefangene von der Wahl allgemein ausschließt, nicht den menschenrechtlichen Mindeststandards genüge. In der Mehrheit der Vertragsstaaten erkannte man Strafgefangenen ein Wahlrecht zu; eine derartig restriktive Regelung wie im Vereinigten Königreich 
stellte eine sehr seltene Ausnahme dar. Die Entscheidung des Gerichtshofs ist im Jahr 2004 mit zwölf zu fünf Richterstimmen ergangen, vom britischen Parlament aber im Jahr 2011 in einer Abstimmung mit 228 zu 20 Stimmen deutlich zurückgewiesen worden. Dies bedeutet, dass der Gerichtshof hier zwar - vielleicht - einen europäischen Konsens richtig bestimmt hat, diesem aber ein offener Dissens in England gegenüberstand.

\section{Problematik der Argumentation des Gerichtshofs}

Lassen Sie mich den Advocatus diaboli spielen und den an der Entscheidung Marckx erläuterten Ansatz des Gerichtshofs zur Bestimmung eines europäischen Konsenses und damit zur Rechtsfortbildung in Europa kritisch hinterfragen. Ich sehe drei wesentliche Einwände:

Erstens beruht die rechtsvergleichende Argumentation im Grunde auf einem problematischen Schluss von einem „normativen Sein“ auf ein „normatives Sollen“. Weil etwas in der Mehrzahl der Mitgliedsstaaten in einer bestimmten Weise geregelt ist, deshalb soll es auch so geregelt sein? Lassen Sie mich ein extremes Beispiel bilden: gäbe es Guantanamos in der Mehrzahl der europäischen Staaten - könnte man aus einem derartigen von den einzelnen Rechtsordnungen tolerierten Ist-Zustand auf einen Soll-Zustand schließen und argumentieren, in Situationen der terroristischen Bedrohung hätten grundlegende Menschenrechtsgarantien zurückzutreten? - Es ist mehr als offensichtlich, dass dies nicht richtig sein kann.

Zweitens ist auch die auf Mehrheiten gestützte Argumentation in Menschenrechtsfragen problematisch. Denken Sie an das Mittelalter - auch wenn die Folter in der Mehrheit oder sogar in allen europäischen Staaten Teil des Strafprozessrechts war, ist von der Tatsache, dass eine bestimmte Anschauung von einer Mehrheit getragen wird, noch nicht auf ihre Richtigkeit zu schließen.

Drittens scheint eine derartige Bestimmung von Standards in Europa auch insofern problematisch zu sein, als die Grenze zwischen Mindest- und Höchststandards verwischt wird. Gehen gesellschaftliche Entwicklungen über eine bestimmte Minimalforderung hinaus, so muss dies nicht bedeuten, dass das bisherige Minimum als nicht mehr tragfähig angesehen wird. In dem erwähnten Fall gegen Deutschland mit der Prozesskostenhilfe für Unternehmen mag es sein, dass einzelne Länder entsprechende Gelder zur Verfügung stellen. Aber muss das sein? Muss der Mindeststandard, nach dem es ausreichend ist, natürlichen Personen in Notfällen einen Anspruch auf willkürfreie Prüfung ihres Anspruchs auf Rechtsdurchsetzung zuzubilligen, aufgegeben, durch einen höheren Standard ersetzt werden? Ist es gerechtfertigt, wenn dann die Gesellschaft für die wirtschaftlichen Ansprüche von Unternehmen in gleicher Weise wie für die Durchsetzung von Individualrechten einstehen muss? Ist überhaupt eine Weiterentwicklung immer eine Verbesserung? Kann sie nicht auch nur eine unter- 
schiedliche Wertung bedeuten, insbesondere in Dreiecksverhältnissen, etwa, wenn es um den Ausgleich von Rechten zwischen Eltern und Kindern oder um klassische Konflikte wie zwischen Persönlichkeitsrecht und Pressefreiheit geht?

\section{Differenzierungen und Einschränkungen}

Dies bedeutet, dass das Argument, es habe sich ein neuer europäischer Konsens herausgebildet und dem habe sich der einzelne Staat nun unterzuordnen, da er andernfalls gegen die Europäische Menschenrechtskonvention verstoßen würde, nur sehr behutsam eingesetzt werden darf. Eine entsprechende Vorsicht charakterisiert auch der Rechtsprechung des Gerichtshofs. Sowohl die Herausarbeitung europäischer Standards als auch die Argumentation auf ihrer Grundlage ist überaus differenziert. Ein Beispiel für eine auf einen neuen europäischen Konsens gestützte Entscheidung wäre etwa das Urteil Bayatyan v. Armenien, in dem es um die Anerkennung eines Kriegsdienstverweigerungsrechts ging. Hier hat der Gerichtshof im Jahr 2011 seine bisherige zurückhaltende Rechtsprechung aufgegeben und mit Blick auf die geänderte Rechtslage in nahezu allen Mitgliedsstaaten als Standard festgehalten, die Weigerung gegen den Dienst mit der Waffe dürfe nicht zu einer Gefängnisstrafe führen. Armenien wurde aufgrund dessen wegen eines Verstoßes gegen Art. 9 EMRK verurteilt.

Häufig ist aber zu beobachten, dass der Gerichtshof seine Argumentation darauf stützt, es lasse sich kein entsprechender neuer europäischer Standard erkennen, daher habe der Staat einen breiten Ermessensspielraum bei der Regelung des Rechtsproblems und eine von den in anderen Staaten gefundenen Lösungen abweichende Lösung stelle keine Konventionsverletzung dar. Dies gilt beispielsweise für die kontroverse Entscheidung Stummer v. Österreich, in der es um die Frage ging, ob Strafgefangene, die arbeiten, in der Rentenversicherung versichert werden müssten. Der Gerichtshof konnte hier, jedenfalls für die streitgegenständliche Zeit, keinen europäischen Konsens erkennen.

Vor diesem Hintergrund ist die nachfolgend dargestellte Entscheidung A, B und C v. Irland von besonderem Interesse, da sie Grenzen der Rechtsfortbildung auch in den Fällen aufzeigt, in denen bereits eine Tendenz zur Herausbildung eines gesamteuropäischen Konsenses zu erkennen ist.

\section{A, B und C v. Irland - Mehrheitsvotum für die Rechtfertigung des Status quo}

Bei der Entscheidung A, B und C gegen Irland geht es um die Abtreibungsproblematik, die in verschiedenen Konstellationen bereits dem Gerichtshof zur Entscheidung vorlag. Während aber in den Entscheidungen Open Door and Dublin Well Woman v. Irland, Vo v. Frankreich und Tysiac v. Polen nur bestimmte Detailfragen - so das Recht auf Information über die Möglichkeit zur Abtreibung im Ausland, die 
Pflicht zum Schutz des Lebens bei einer versehentlich durchgeführten Abtreibung und die verfahrensmäßige Absicherung der Durchführung einer medizinisch indizierten Abtreibung - thematisiert wurden, betrifft die Beschwerde von A, B und C das auf verfassungsrechtlicher Ebene verankerte Verbot der Abtreibung in Irland als Grundsatzproblem. Antragstellerinnen sind drei Frauen, die in Großbritannien eine Abtreibung vorgenommen haben, da ihnen die entsprechende Möglichkeit in Irland nicht zur Verfügung stand, eine Abtreibung im Ausland aber nach irischem Recht straffrei möglich war. Sie alle beschweren sich wegen einer Vielzahl von Menschenrechtsverletzungen, konkret von Art. 2, 3, 8, 13 und 14 EMRK.

Bei der Antragstellerin C liegt eine medizinisch indizierte Abtreibung vor, da sie an Krebs erkrankt ist und Angst vor einer Schädigung des Fötus und vor einer Gefährdung ihrer eigenen Gesundheit hat. In diesem Fall moniert der Gerichtshof einstimmig eine Verletzung der Konvention aufgrund der unzureichenden Schutzregelungen in Irland.

Problematisch sind dagegen die Beschwerden der Antragstellerinnen A und B. Die erste Antragstellerin ist eine alkoholkranke Frau, die bereits vier Kinder hat und erneut gegen ihren Willen schwanger wird. Ihren Wunsch nach Abtreibung begründet sie damit, dass sie andernfalls die Chance, ihre in Pflege untergebrachten Kinder wiederzubekommen gefährde, da aufgrund einer zu erwartenden postnatalen Depression ein Rückfall in die Alkoholabhängigkeit zu erwarten sei. Die zweite Antragstellerin hat Furcht, dass besondere Komplikationen bei der Schwangerschaft bestehen könnten. Allerdings ist bereits vor der Abtreibung medizinisch erwiesen, dass es keine besondere Gefährdung gibt. Die Notwendigkeit, die Abtreibung im Ausland vorzunehmen, hat für beide Antragstellerinnen zusätzliche gesundheitliche Probleme zur Folge.

Der Gerichtshof sieht lediglich eine Verletzung von Art. 8 EMRK als diskussionswürdig an, verneint diese aber im Ergebnis mit 11 zu 6 Stimmen.

Die Auseinandersetzung um die Frage, ob sich ein Recht auf Abtreibung entwickelt haben könnte, zeigt wie ein Brennspiegel die Problematik der Grenzziehung bei der rechtsfortbildenden Auslegung des Grundrechtsschutzes

Fraglich ist, ob der Nachweis einer Tendenz zur Konvergenz der nationalen Abtreibungsgesetze in Europa ausreichend sein kann, um daraus die Schlussfolgerung zu ziehen, dass der nationale Gestaltungsspielraum entscheidend eingeschränkt und ein Sonderweg Irlands damit nur mehr schwer begründbar ist. Die irische Regierung setzt bei ihrer Argumentation daran an, dass eine entsprechende „Harmonisierung“ die „anerkannte Bedeutung und fundamentale Rolle des demokratischen Prozesses in 
jedem Staat und die von Art. 53 EMRK garantierte Diversität der Traditionen und Wertsetzungen in den Vertragsstaaten “ gefährden würde. ${ }^{8}$

Umstritten ist allerdings zunächst, ob eine Konvergenz der nationalen Abtreibungsgesetze überhaupt erkennbar sei. In drei europäischen Staaten - San Marino, Malta und Andorra - ist die Gesetzgebung noch restriktiver als in Irland und schließt straffreie Abtreibungen ausnahmslos aus. Allerdings ist eine Abtreibung aus sozialen Gründen in etwa 35 Vertragsstaaten grundsätzlich möglich. Zudem ist eine Liberalisierung des Abtreibungsrechts in Monaco, Montenegro, Portugal und Spanien zu beobachten. Der Befund ist damit nicht eindeutig, aber weniger vage als etwa in der Marckx-Entscheidung. Der Gerichtshof geht daher auch, anders als die irische Regierung, von der Herausbildung eines entsprechenden europäischen Konsenses aus ohne eine Analyse der völkerrechtlichen Rechtsentwicklung für nötig zu halten.

Dennoch - und hier zeichnet sich in der Entwicklung des case law eine entscheidende Wende ab - erklärt der Gerichtshof entgegen der allgemeinen Regel, nach der bei einem sich abzeichnenden Konsens der verschiedenen nationalen Gesetzgebungen der „margin of appreciation“ eingeschränkt ist, dass der beobachtete Konsens den weiten Entscheidungsspielraum des nationalen Gesetzgebers in diesem Fall nicht entscheidend schmälere:

"However, the Court does not consider that this consensus decisively narrows the broad margin of appreciation of the State."

Hauptargument des Gerichtshofs ist, dass man den Zeitpunkt der Entstehung menschlichen Lebens auf der Grundlage der Konvention nicht mit juristischen Mitteln eindeutig feststellen könne. Daher sei nicht auszuschließen, dass sich das aus Art. 2 EMRK ableitbare Recht des Kindes auf Leben und die aus Art. 2 und 8 EMRK ableitbaren Rechte der schwangeren Mutter grundsätzlich unvereinbar gegenüberstünden. Bei einem derartigen Grundrechtskonflikt könne aber eine Tendenz zur Herausbildung eines einheitlichen Ansatzes in verschiedenen nationalen Rechtsordnungen nicht der entscheidende Faktor sein, um einen gerechten Ausgleich zwischen den verschiedenen Rechten und Interessen zu finden. Und dies, so betont der Gerichtshof, gelte auch trotz der evolutiven Interpretation der Konvention.

Dies ist eine eindeutige Aussage des Gerichtshofs: Geht es um den Ausgleich zwischen verschiedenen, von der Konvention gleichermaßen geschützten Rechten, besteht für die Vertragsstaaten kein „Zugzwang“, sich dem von der Staatenmehrheit favorisierten Modell anzuschließen. Vielmehr lässt diese Konstellation wieder Raum für individuelle, vom gesellschaftlichen Konsens im jeweiligen Land getragene Lösungen und dies selbst dann, wenn sie von den in anderen Ländern gefundenen Lösungen - wie 
gerade beim irischen Abtreibungsrecht - grundlegend abweichen. In diesen Fällen enthält sich der Gerichtshof, anders als im Fall Marckx, auch einer Stellungnahme zu dem sozialpolitisch Wünschbaren und Vernünftigen und verweist die Frage an den nationalen Gesetzgeber zurück:

„As noted above, by reason of their direct and continuous contact with the vital forces of their countries, the State authorities are, in principle, in a better position than the international judge to give an opinion, not only on the "exact content of the requirements of morals" in their country, but also on the necessity of a restriction intended to meet them. ${ }^{10}$

Die dissentierenden Richter dagegen argumentieren streng auf der Basis der MarckxRechtsprechung, gehen von einem "unzweifelhaft starken Konsens unter den europäischen Staaten" mit Blick auf einen Vorrang der Rechte der schwangeren Frau gegenüber den Rechten des ungeborenen Kindes aus und bekennen sich explizit auch bei derartigen Wertungskonflikten zwischen verschiedenen Grundrechtspositionen - zu einer harmonisierenden Rolle der Rechtsprechung des Gerichtshofs. Damit wird letztlich einem gewissen Automatismus bei der Rechtsfortbildung der Grund- und Menschenrechte das Wort geredet. Die Mehrheit der Richter und Richterinnen des Gerichtshofs lehnt dies ab, nimmt stattdessen eine Einzelfallabwägung vor und akzeptiert einen „moralischen Vorbehalt“ der Vertragsstaaten in besonders sensiblen Grundrechtsfragen. Die Minderheit des Gerichtshofs stellt sich dem entgegen:

„... it is the first time that the Court has disregarded the existence of a European consensus on the basis of "profound moral views". Even assuming that these profound moral views are still well embedded in the conscience of the majority of Irish people, to consider that this can override the European consensus, which tends in a completely different direction, is a real and dangerous new departure in the Court's case-law. A case-law which to date has not distinguished between moral and other beliefs when determining the margin of appreciation which can be afforded to States in situations where a European consensus is at hand." 11

In den Augen der Mehrheit, die die Entscheidung trägt, wird aber gerade an diesem Punkt die Grenze der richterlichen Rechtsfortbildung erreicht. Die Rolle des Gerichtshofs bei der Durchsetzung menschenrechtlichen Standards wird nicht in einer Angleichung nach „oben“ - wo auch immer „oben“ sein mag - gesehen, sondern Rozakis, Tulkens, Fura, Hirvelä, Malinverni und Poalelungi,,Rd. 9. 
erneut auf die Wahrung von allgemein akzeptierten (Mindest)standards zurückgebunden.

Dies bedeutet, dass die Feststellung eines „europäischen Konsenses“ auf der Grundlage rechtsvergleichender Analysen sowie mit Blick auf Weiterentwicklungen im Völkerrecht und Stellungnahmen der Organe des Europarats nur Indizwirkung für ein gewandeltes Grundrechtsverständnis haben, die Auslegung der Konvention und damit das Urteil über das Vorliegen einer Konventionsverletzung aber nicht unmittelbar bestimmen kann. Denn das „menschenrechtliche Sollen“ kann auch im Widerspruch zu den von der Mehrheit der Staaten akzeptierten Normen stehen. Die Konvention, so ist aus der Entscheidung ABC v. Irland zu entnehmen, muss nicht immer Veränderungen bewirken und in die Richtung des mainstream " weisen. Auch dem „mainstream“ entgegen gesetzte Lösungen können für akzeptabel gehalten werden.

\section{F. Ausblick}

Der Europäische Gerichtshof für Menschenrechte hat sich in Europa - im Zusammenspiel mit den nationalen Verfassungsgerichten - als Motor für den behutsamen, evolutionären, aber dennoch deutlich sichtbaren Wandel der Grund- und Menschenrechte und damit der in Europa anerkannten Grundwerte gezeigt. Die vielschichtigen und oftmals kontroversen Entscheidungen des Gerichtshofs machen deutlich, wie notwendig es ist, den Facetten des Wandels detailgenau nachzuspüren. Die Grenzen einer dynamischen Grundrechtsinterpretation können nicht a priori definiert werden; europäische Standards können nicht von oben „verordnet“ werden. Die Rechtsprechung des Gerichtshofs muss Sensor für Grundrechtsgefährdungen ebenso wie für mögliche Lösungsansätze sein, und dies, ohne die von Montesquieu in seinem „Esprit des lois“ beschriebenen Unterschiede in „Gemeingeist, Sitten und Lebensstil“ in den vielgestaltigen europäischen Staaten geringzuschätzen. 